\title{
Optical Orientation of Trions in Charge-Tunable InAs/GaAs Quantum Dots
}

\author{
S. Laurent ${ }^{a}$, B. $\operatorname{EBlE}^{a}, \mathrm{O} \mathrm{KReBS}^{a}, \mathrm{~A} . \mathrm{Lemaître}^{a}$,

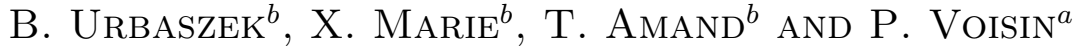 \\ ${ }^{a}$ Laboratoire de Photonique et Nanostructures-CNRS \\ Route de Nozay, 91460 Marcoussis, France \\ ${ }^{b}$ Laboratoire de Magnétisme, Nanophysique et Optoélectronique, INSA \\ 135 Avenue de Rangueil, 31077 Toulouse Cedex, France
}

\begin{abstract}
We report on optical orientation of excitons and trions (singly charged exciton) in individual charge-tunable self-assembled InAs/GaAs quantum dots. When the number of electrons varies from 0 to 2 , the trion photoluminescence under quasi-resonant excitation gets progressively polarized from zero to $\approx 100 \%$. We discuss this behavior as the efficient quenching of exciton spin quantum beats in anisotropic quantum dots due to the trion formation. This result indicates a long hole-spin relaxation time larger than the radiative lifetime, confirmed by time-resolved photoluminescence measurements carried out on a quantum dots ensemble.
\end{abstract}

PACS numbers: 71.70.-d, 72.25.Fe, 73.21.La, 78.47.+p

\section{Introduction}

Schottky diodes containing a layer of self-assembled quantum dots (QDs) close to the $n^{+}$-electrode have been recently used for tuning the number of resident electrons in QDs by applying a gate voltage [1-5]. In the context of using electrons trapped in QDs for quantum information processing, such systems appear very promising. As, in contrast to chemical doping, the QD charge can be precisely tuned, they offer a unique way to investigate the spin relaxation of carriers trapped in QDs and their mutual spin-dependent interaction. For example, in a trion complex (i.e., 2 electrons +1 hole confined in a dot), the exchange 
interaction vanishes in the ground state which removes the energy splitting of excitons between optically allowed and forbidden states as well as the fine structure of bright excitons observed in elongated QDs [6,7]. The circular polarization of a negative (positive) trion photoluminescence (PL) should in principle reflect the spin state of the hole (electron) as already reported in [5], giving access to intrinsic spin relaxation mechanisms.

Here, we report on optical orientation experiments under quasi-resonant excitation of individual charge-tunable QDs embedded in the intrinsic part of a $n$ - $i$-Schottky diode. We focus on the negative trion luminescence and show that its circular polarization strongly depends on the QD equilibrium charge $(0,1$, or 2$)$ before the excitation. We discuss these observations as the consequence of holespin precession in the anisotropic exchange field during the trion relaxation time which depends on the applied electric bias. Time-resolved optical orientation of a QD ensemble supports our interpretation, and directly demonstrates the hole-spin relaxation quenching in a trion complex.

\section{Experimental}

We studied two samples grown using molecular-beam epitaxy on a [100]-oriented semi-insulating GaAs substrate. The field-effect structure consists of a $200 \mathrm{~nm}$ thick $n^{+}$-GaAs layer, followed by $25 \mathrm{~nm}$ of intrinsic GaAs, $\approx 2$ monolayers of strained InAs, $30 \mathrm{~nm}$ of GaAs, $100 \mathrm{~nm}$ of $\mathrm{Al}_{0.3} \mathrm{Ga}_{0.7} \mathrm{As}$ acting as a blocking barrier and, finally, a $20 \mathrm{~nm}$ GaAs cap layer. The density of the QDs grown in the Stranski-Krastanow mode is estimated to be less than $10^{9} \mathrm{~cm}^{-2}$ in sample A designed for single QD spectroscopy and a few $10^{10} \mathrm{~cm}^{-2}$ in sample B. To control the internal built-in electric field and thus the QD excess charge, electrodes were patterned on the surface of the samples by optical lithography. The "back" electrode contacting the $n^{+}$-GaAs layer is fabricated by annealing a $4000 \AA$ multilayer of $\mathrm{Ni} / \mathrm{Ge} / \mathrm{Au}$. The Schottky contact allowing optical investigation consists in a semi-transparent $50 \AA \mathrm{Ni}_{0.5} \mathrm{Au}_{0.5}$ film. For individual QD spectroscopy latex micro-spheres were deposited, subsequently covered by a $2000 \AA$ Au layer and then removed, providing optical apertures of $1 \mu \mathrm{m}$ diameter through an opaque metallic mask.

\section{Results and discussion}

The capacitance-voltage profile shown in Fig. 1 was measured at low temperature $(\approx 10 \mathrm{~K})$ and in darkness to determine the voltage regime of QD charging. This technique was sensitive enough only for sample B. When the gate voltage $V_{\mathrm{g}}$ varies from $-200 \mathrm{mV}$ to $+350 \mathrm{mV}$, the capacitance increases by steps reflecting the successive filling of quantized states. The ground state filling corresponds to the first step, here at $V_{\mathrm{g}} \approx 0 \mathrm{~V}$ in a fair agreement with the electron confinement energy of $\approx 150 \mathrm{meV}$ below the GaAs conduction band edge and a Schottky barrier 


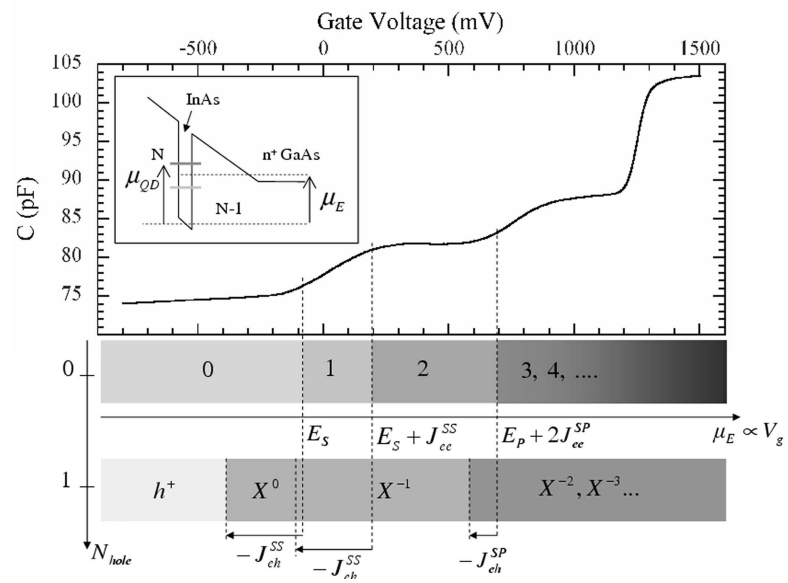

Fig. 1. $C-V$ characteristic of a Schottky diode containing a single InGaAs/GaAs QD layer of large density (sample B). The bottom diagrams show the domain of QD charging as a function of the electrode chemical potential $\mu_{\mathrm{E}}$ without illumination $\left(N_{\text {hole }}=0\right)$ and after the capture of a photocreated hole denoted $h^{+}$.

of $0.8 \mathrm{eV}$. Yet, in contrast to other similar studies $[2,8,3]$, we could not resolve here the Coulomb blockade splitting $\left(J_{\mathrm{eh}}^{S S} \approx 30 \mathrm{meV}\right.$ or $\left.\Delta V_{\mathrm{g}} \approx 200 \mathrm{mV}\right)$ between 1 and 2 electrons occupying the $S$-orbital ground state of the QD. This is probably due to the large inhomogeneous distribution of ground state energies estimated by PL experiments to be $\approx 50 \mathrm{meV}$. Bottom part of Fig. 1 shows the resulting diagram of electron occupation for a single QD. The charging from $N-1 \rightarrow N$ occurs when the potential $\mu_{\mathrm{E}}$ of the $n^{+}$-electrode reaches the QD chemical potential $\mu_{\mathrm{QD}}(N)$. The latter can be seen as the single particle energy of one electron (measured from the QD bottom) in the QD charged with $N-1$ electrons [9]. Similarly, when the dot has captured a photo-created hole under optical excitation, we can deduce the stability diagram of the charged excitons $X^{-n}$. To the first order, the domains are simply shifted by the direct electron-hole Coulomb interaction $\left(J_{\mathrm{eh}}^{S S}\right.$ or $\left.J_{\mathrm{eh}}^{S P}\right)$ as shown in Fig. 1. A remarkable feature of this diagram is the wide stability domain of the trion complex $X^{-}$as a consequence of the large separation between $S$ and $P$ electron levels $(\approx 60 \mathrm{meV})$. Hence, the trions form within a voltage range where the QD equilibrium occupation is either 0 (on a narrow range), 1 or 2 , but with a resulting dynamics that should depend on this initial charge as discussed in the following.

We used a tunable cw Ti:sapphire laser for the PL excitation and a confocal experimental arrangement based on a $\times 50$ microscope objective providing the required spatial resolution. The samples were attached to the cold finger of a liquid He flux cryostat assuring a temperature $<20 \mathrm{~K}$. The circular polarization of the laser beam was set to $\sigma^{+}$by a quarter-wave quartz plate, and the PL polarization $\sigma^{+}$or $\sigma^{-}$was analyzed by a quarter-wave birefringent filter placed 
in front of a fixed Glan-Thomson linear polarizer. Of crucial importance is the polarization-unsensitive beamsplitter required to separate the emitted PL from the incident optical path. The whole setup provided a rejection ratio of 100:5 for crossed polarizations $\left(I_{\sigma^{+} \sigma^{+}}: I_{\sigma^{+} \sigma^{-}}\right)$over the spectral region of interest. The collected luminescence was then spectrally filtered by a $0.6 \mathrm{~m}$ focal length double monochromator and detected by a liquid-N cooled CCD array. The high spectral resolution of our setup $(40 \mu \mathrm{eV})$ allowed us to measure line shifts as small as $15 \mu \mathrm{eV}$.

We report here results performed under quasi-resonant (or intra-dot) excitation, the laser energy being fixed $70 \mathrm{meV}$ above the detection. The circular polarization $P_{\text {circ }}$ is defined as usually by $P_{\text {circ }}=\left(I_{\sigma^{+} \sigma^{+}}-I_{\sigma^{+} \sigma^{-}}\right) /\left(I_{\sigma^{+} \sigma^{+}}+I_{\sigma^{+} \sigma^{-}}\right)$. Under these conditions optical excitation and recombination both involve valence states belonging essentially to the heavy-hole band [10], which should give rise to positive circular polarization. Figure 2a shows typical polarization-resolved PL
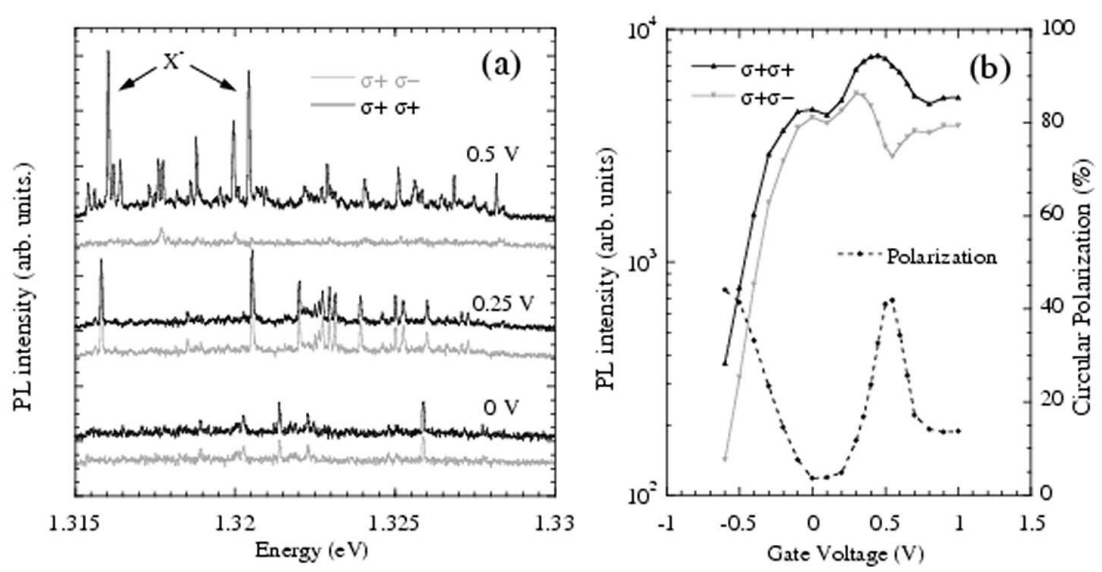

Fig. 2. (a) Polarization-resolved spectra of sample A excited at $1.37 \mathrm{eV}$ without optical mask for different gate voltages. Curves are shifted vertically for clarity. (b) PL and polarization of a QD ensemble (sample B) under excitation at $1.34 \mathrm{eV}$ against gate voltage. The signal is averaged over a detection window of $1 \mathrm{meV}$ around $1.27 \mathrm{eV}$.

spectra of a small set of QDs (sample A without optical mask) at different gate voltages and with a power density of $\approx 100 \mathrm{~kW} \mathrm{~cm}{ }^{-2}$. It illustrates a very remarkable behavior: for bias below $300 \mathrm{mV}$ the PL polarization is basically zero but increases strongly with the bias, up to $+100 \%$ at $500 \mathrm{mV}$ for most of the lines. For this bias, the quantum dots are filled with two electrons, so that the narrow PL lines correspond most probably to optical emission of trions. Their polarization is thus a signature of the hole spin along the optical axis, while their full polarization reveals a spin relaxation much longer than the radiative lifetime $\tau_{\mathrm{r}} \approx 1 \mathrm{~ns}$, as already reported for $n$-doped InAs QDs [11] and CdSe QDs [5]. Sam- 
ple B shows a similar behavior, albeit smoothened by the large number of QDs, with a maximum polarization of $42 \%$ at $550 \mathrm{mV}$. These preliminary observations provide a rough statistical description of the voltage-dependence of the PL polarization, from which a given individual QD can deviate because of different size, shape anisotropy, composition, or coupling to the electrode. Besides, the polarization analysis does not suffer here from the passing through a $1 \mu \mathrm{m}$ size aperture, which provides a good basis to discuss measurements on single QDs.

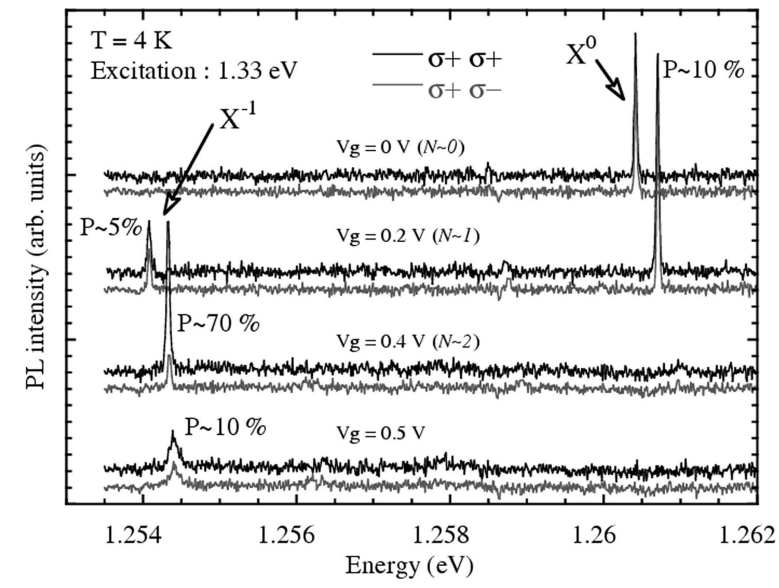

Fig. 3. Polarization-resolved PL spectra of a single QD for different bias. The trion feature appearing $6.7 \mathrm{meV}$ below the exciton line appears with a weak polarization and gets strongly polarized at $0.4 \mathrm{~V}$.

Figure 3 shows the polarization evolution of an individual QD that represents well the typical encountered behaviors. The $X^{0}$ neutral exciton is weakly polarized like the trion feature $X^{-}$which appears $6.7 \mathrm{meV}$ below at $V_{\mathrm{g}}=0.2 \mathrm{~V}$. The latter gets however rapidly polarized up to $70 \%$ when increasing the bias, and then above $0.4 \mathrm{~V}$ starts broadening while its polarization simultaneously collapses. The maximum polarization of every single trion line we measured never reached $100 \%$, lying most of the time in the 50-80\% range, unlike the QDs studied without optical mask (Fig. 2). We attribute this discrepancy to the $\mu \mathrm{m}$ size aperture which partially destroys the optical polarization. Nevertheless our single QD measurements clearly evidence that the trion polarization depends on the bias, i.e. on the QD equilibrium occupation $N$. The maximum is reached when $X^{0}$ has completely disappeared, hence for $N=2$. The precise intra-dot excitation process is not clearly identified but did not turn out to be critical in order to observe this effect. Therefore we believe that the excitation mainly involves transitions between a confined hole state and the conduction quasi-2D continuum. The hole relaxes rapidly with a well defined spin while the photoelectron rapidly tunnels instead towards the $n^{+}$-electrode in a few ps. The average time of trion formation 
is then governed by the bias-dependent coupling with this reservoir of electrons. Clearly, it can be continuously tuned from a few ps when the dot already contains 2 electrons $(N=2)$ up to a few ns when the trion and neutral exciton features coexist $\left(N=0^{+}\right)$. During this thermalization, the hole spin can relax under the anisotropic exchange interaction (AEI) with electrons as we explain now. AEI is responsible in anisotropic QDs for the splitting of the bright excitons with angular quantum number $j= \pm 1$ into linearly polarized eigenstates $|x\rangle,|y\rangle$. The energy splitting $\delta_{1}$ amounts to a few tens $\mu \mathrm{eV}$ for the InAs/GaAs system, and in our case was large enough to be measured in $\approx 1 / 3$ of the investigated single QDs with values from $30 \mu \mathrm{eV}$ to $80 \mu \mathrm{eV}$. The AEI gives rise to quantum beat oscillations of excitons photocreated as a coherent superposition of $|x\rangle$ and $|y\rangle$ (as observed
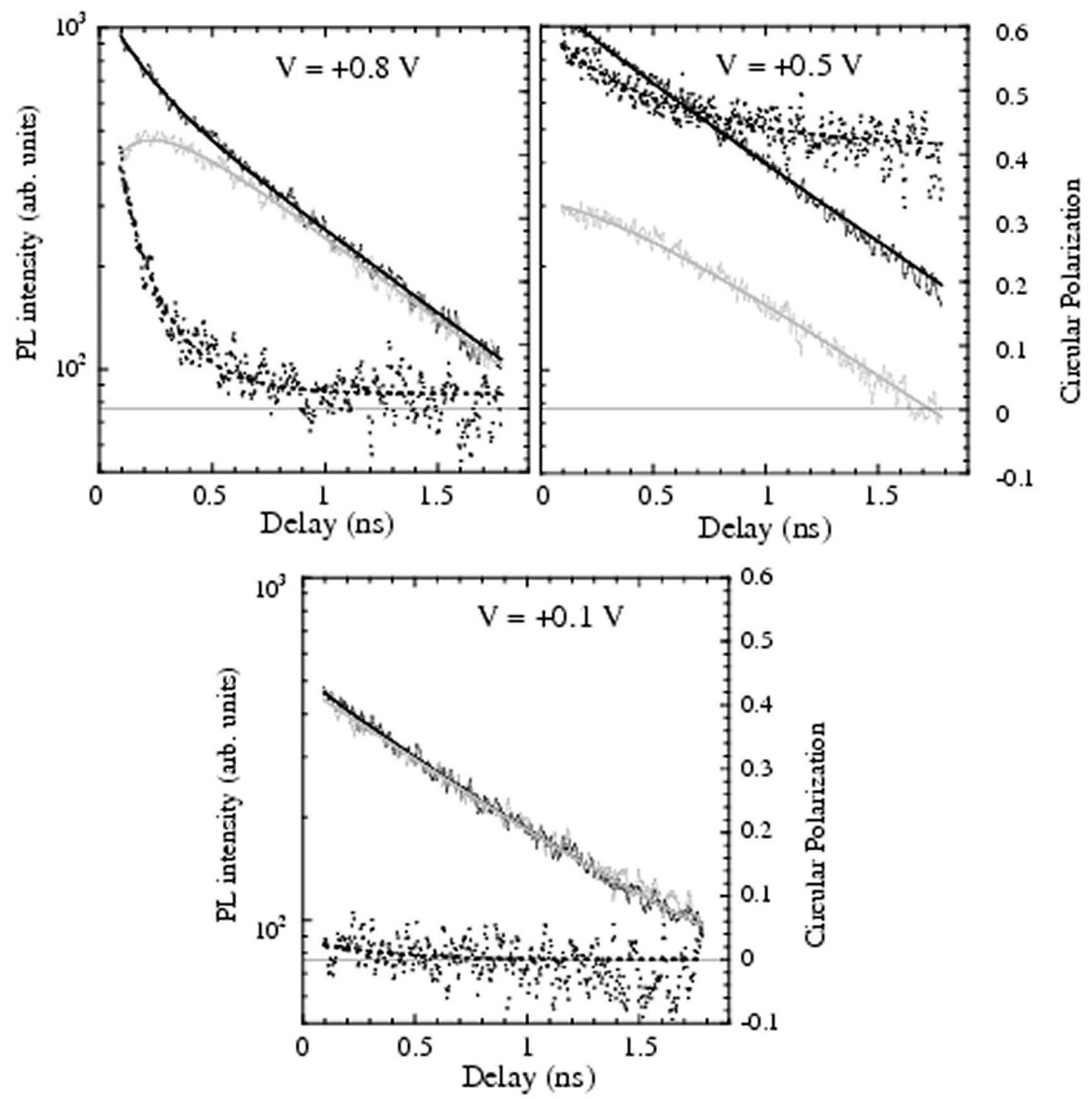

Fig. 4. Time-resolved PL intensity (solid lines) in $\sigma^{+} \sigma^{+}$(black) and $\sigma^{+} \sigma^{-}$(gray) configurations, and circular polarization (black symbols) of sample B excited at $1.34 \mathrm{eV}$. Profiles are fitted by (multi)-exponential decays. 
in Refs. $[12,13])$, with a period $\tau_{\mathrm{AEI}}=h / \delta_{1}$ falling into the $5-100$ ps range. This effect prevents observation of a sizeable PL circular polarization of excitons in time-integrated experiments. For a trion in its ground state (i.e., $2 S$-electrons, $1 S$-hole), the electron-hole exchange interaction vanishes because of the 2-electron singlet configuration. Therefore, as soon as the trion is thermalized in the QD the hole-spin precession in the anisotropic exchange field is quenched. To the first order, if $\tau_{X^{-}}$is greater than some effective precession period $\tau_{\text {AEI }}^{*}$ the resulting hole-spin polarization is averaged to zero, but gets maximum when $\tau_{X^{-}} \ll \tau_{\text {AEI }}^{*}$. To support this interpretation and in particular that the trion spin orientation is frozen once thermalized, we performed time-resolved spectroscopy of sample B. A standard macro-PL setup equipped with a streak-camera synchronized to a mode-locked ps Ti:Sa laser for the excitation was used providing a time resolution of 50 ps. Figure 4 gives a rapid glimpse of our results. At $+0.1 \mathrm{~V}$ the PL of excitons is basically unpolarized during all the recombination (for delays above $100 \mathrm{ps}$ ), while trion PL shows at $+0.5 \mathrm{~V}$ a high degree of polarization with a component which does not relax within our temporal window $\left(\tau_{\text {spin }}>20 \mathrm{~ns}\right)$. At higher bias, the initial polarization remains high, as a signature of the photocreated hole spin, but damps rapidly in $\approx 200$ ps probably because of nonzero AEI in multi-charged excitons.

\section{Conclusions}

We have performed optical orientation of trions in single charge-controlled InAs QDs. The expected quenching of electron-hole exchange interaction turns out to be strongly dependent on the bias, which we attribute to a peculiar trion thermalization process. Time-resolved measurements of PL polarization give a 20 ns lower limit for the intrinsic hole-spin relaxation time, making it an alternative candidate to the electron spin for quantum information processing in QDs.

\section{References}

[1] H. Drexler, D. Leonard, W. Hansen, J.-P. Kotthaus, P.M. Petroff, Phys. Rev. Lett. 73, 2252 (1994).

[2] R.J. Warburton, C.S. Dürr, K. Karrai, J.P. Kotthaus, G. Medeiros-Ribeiro, P.M. Petroff, Phys. Rev. Lett. 79, 5282 (1997).

[3] J.J. Finley, P.W. Fry, A.D. Ashmore, A. Lemaître, A.I. Tartakovskii, R. Oulton, D.J. Mowbray, M.S. Skolnick, M. Hopkinson, P.D. Buckle, P.A. Maksym, Phys. Rev. B 63, 161305 (2001).

[4] B. Urbaszek, R.J. Warburton, K. Karrai, B.D. Gerardot, P.M. Petroff, J.M. Garcia, Phys. Rev. Lett. 90, 247403 (2003).

[5] T. Flissikowski, I.A. Akimov, A. Hundt, F. Henneberger, Phys. Rev. B 68, 161309 (2003). 
[6] M. Bayer, G. Ortner, O. Stern, A. Kuther, A.A. Gorbunov, A. Forchel, P. Hawrylak, S. Fafard, K. Hinzer, T.L. Reinecke, S.N. Walck, J.P. Reithmaier, F. Klopf, F. Schäfer, Phys. Rev. B 65, 195315 (2002).

[7] K.V. Kavokin, Phys. Rev. B 69, 75302 (2004).

[8] R.J. Luyken, A. Lorke, A.O. Govorov, J.P. Kotthaus, G. Medeiros-Ribeiro, P.M. Petroff, Appl. Phys. Lett. 74, 2486 (1999).

[9] A. Wojs, P. Hawrylak, Phys. Rev. B 53, 10841 (1996).

[10] G. Bester, S. Nair, A. Zunger, Phys. Rev. B 67, 161306 (2003).

[11] M. Senes, X. Marie, B. Liu, T. Amand, O. Krebs, P. Voisin, J.M. Gerard, in: Proc. 26th Conf. on the Physics of Semiconductors, Edinburgh 2002, Institute of Physics Conference Series 171, Eds. A.R. Long, J.H. Davies, Institute of Physics, Bristol 2003, H233.

[12] T. Flissikowski, A. Hundt, M. Lowisch, M. Rabe, F. Henneberger, Phys. Rev. Lett. 86, 3172 (2001).

[13] A.S. Lenihan, M.V. Gurudev Dutt, D.G. Steel, S. Ghosh, P.K. Bhattacharya, Phys. Rev. Lett. 88, 223601 (2002). 